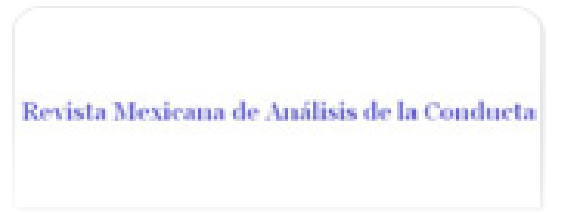

Revista Mexicana de Análisis de la Conducta ISSN: 0185-4534

editora@rmac-mx.org

Sociedad Mexicana de Análisis de la Conducta México

RAMOS PRADO, ILIANA GUADALUPE; SANTOYO VELASCO, CARLOS

Organización y estabilidad del comportamiento coercitivo en niños escolares: una perspectiva de desarrollo

Revista Mexicana de Análisis de la Conducta, vol. 34, núm. 2, diciembre, 2008, pp. 265-292

Sociedad Mexicana de Análisis de la Conducta

Guadalajara, México

Disponible en: http://www.redalyc.org/articulo.oa?id=59311115008

- Cómo citar el artículo

- Número completo

- Más información del artículo

Página de la revista en redalyc.org

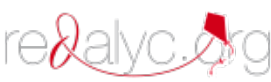

Sistema de Información Científica

Red de Revistas Científicas de América Latina, el Caribe, España y Portugal Proyecto académico sin fines de lucro, desarrollado bajo la iniciativa de acceso abierto 


\title{
ORGANIZACIÓN Y ESTABILIDAD DEL COMPORTAMIENTO COERCITIVO EN NIÑOS ESCOLARES: UNA PERSPECTIVA DE DESARROLLO'
}

\author{
ORGANIZATION AND STABILITY OF COERCIVE BEHAVIOR \\ IN ELEMENTARY SCHOOLS CHILDREN: A DEVELOPMENTAL \\ PERSPECTIVE
}

\section{ILIANA GUADALUPE RAMOS PRADO Y CARLOS SANTOYO VELASCO UNIVERSIDAD NACIONAL AUTÓNOMA DE MÉXICO}

\begin{abstract}
RESUMEN
La perspectiva de desarrollo postula que la organización del comportamiento es producto de la interacción recíproca y dinámica con la ecología social, destacando factores situacionales que afectan el comportamiento subsecuente. Se examina el efecto que tienen factores conductuales, cognoscitivos y grupales sobre patrones de comportamiento coercitivo en niños Riesgo. Diez niños Riesgo y diez de un grupo de Comparación acoplado de tercer grado de primaria, fueron seguidos en dos cortes anuales mediante una estrategia multi-método. El análisis de los datos indica que los niños Riesgo se diferencian de los niños Control en aspectos conductuales, cognoscitivos y grupales. Algunos niños Riesgo son centrales en la red social, y los profesores los perciben como conflictivos en la escuela. Los eventos de provocación funcionan en los niños Riesgo como estímulos que facilitan episodios coercitivos, mientras que en los niños del grupo de Comparación, dichos eventos son estímulos inhibitorios. Las interacciones de los niños Riesgo en la escuela, pueden consolidar patrones de comportamiento coercitivo. La relación con el
\end{abstract}

1. El trabajo forma parte de la tesis doctoral de Ramos Prado bajo la dirección del segundo autor, el cual agradece el apoyo de CONACYT al proyecto 057327. Para comunicarse con los autores escribir a jriscor@yahoo.com.mx y/o carsan@servidor.unam.mx.

Recibido: 27 de Junio, 2008. Revisado: 27 de Octubre, 2008. Aceptado: 10 de Diciembre, 2008. 
profesor, el ambiente coercitivo y punitivo, y las relaciones con sus pares son centrales en la consolidación de patrones diferenciales.

Palabras Claves: Patrón de Comportamiento Coercitivo, Coerción, Interacción Social, Eventos Disposicionales.

\begin{abstract}
Developmental perspective postulates that behavior organization is the result of the reciprocal and dynamic interaction with their social ecology, emphasizing situational factors which affect subsequent behavior. This paper examines the effect of behavioral, cognitive and group factors on behavioral patterns of coercive on-risk children. Ten elementary school on-risk children and ten matched controls from third grade were followed for two years with a multi-method strategy. Data analysis indicates that on-risk children differ from matched children in behavioral and cognitive aspects, and in their social networks. Some on-risk children are central in the social network, and teachers perceive them as more conflictive than matched children. For on-risk children, provocations are facilitating stimulus of coercive episodes, whereas for matched children these events were inhibitory stimulus. For on-risk children, school interaction may consolidate patterns of coercive behavior. Teachers' perception, coercive and punitive environment and social relations with peers are central in the consolidation of differential patterns.
\end{abstract}

Key Words: Pattern Coercive Behavior, Coercion, Social Interaction, Setting Events.

Los patrones de comportamiento antisocial comprenden a la agresión, el vandalismo, la infracción de reglas, el desafío de autoridad, la violación de normas sociales, entre otras modalidades conductuales. En la escuela, el comportamiento antisocial de los niños toma diferentes formas como: robo a los pares, abandonar la escuela, desafiar a la autoridad, tener pares con características antisociales y mostrar poco involucramiento en las actividades escolares.

Esas manifestaciones asociadas a diversos factores del contexto pueden determinar la organización del comportamiento antisocial; por ejemplo, los niños coercitivos tienden a relacionarse con pares que apoyen o complementen su comportamiento coercitivo.

Por ello, para estudiar la agresión se deben considerar interacciones específicas en el medio natural e identificar aquellos factores sociales que favorecen su ocurrencia.

En este trabajo, la agresión se define como un subconjunto de actos coercitivos empleados por los miembros de una relación, para alterar el com- 
portamiento del otro (Patterson, 1982). Es así, que la estructura de los conflictos diádicos se considera como una unidad de análisis adecuada para identificar cómo las interacciones coercitivas son establecidas, mantenidas y organizadas; partiendo del supuesto de que el cambio o la continuidad de los patrones de comportamiento coercitivo dependen de factores proximales de interacción. Así, la coerción no depende exclusivamente de rasgos de personalidad sino de la clase de situaciones en las que ésta se presenta (Santoyo, 1997). Las personas, al interactuar con su ambiente representan un estímulo para los otros, lo que propicia la emisión de diversos comportamientos. Así, por ejemplo, es factible identificar estímulos sociales sistemáticos que anteceden al comportamiento coercitivo; específicamente aquellas clases de estímulos que se asocian con el aumento o disminución de la probabilidad de control de las respuestas de otros; eventos que inhiben, facilitan o aceleran el comportamiento coercitivo en los niños (Patterson, 1974). Si bien el enfoque de coerción de Patterson (1982) supone que la persona coercitiva exhibirá una alta frecuencia de eventos aversivos a sus congéneres no debemos dejar de lado que estos niños pueden también exhibir actos aversivos ante provocaciones o estimulación aversiva que otros les dirigen, siendo el mecanismo de control, en este caso, regulado por el reforzamiento negativo (Sidman, 1989).

Por otra parte, desde el punto de vista de la Teoría de la Naturaleza Humana de Elección Conductual, TNHEC (Wilson \& Herrnstein, 1985) se puede contribuir al estudio del comportamiento coercitivo si se entiende como un proceso de elección. Por lo tanto, desde esa perspectiva es conveniente desarrollar estudios empíricos que den cuenta del proceso de elección para explicar patrones diferenciales de comportamiento coercitivo.

De esta forma, se pretende integrar explicaciones como las que se derivan de la TNHEC en el ámbito escolar junto con la perspectiva de desarrollo. El modelo de la TNHEC supone que la vida cotidiana representa un continuo de elecciones, las cuáles están determinadas por sus consecuencias. Así, se espera que los cursos de acción elegidos sean los que aportan las consecuencias más preferibles para la persona. De esta forma, el niño compara los beneficios y las pérdidas de elegir comportamiento coercitivo o de no elegirlos. Para los niños Riesgo, el comportamiento coercitivo puede ser reforzante porque su elección implica beneficios inmediatos. Por el contrario, este mismo comportamiento puede debilitarse si los pares y/o el profesor promueven comportamientos sociales y/o académicos en el aula.

Por ejemplo, en el escenario escolar Wilson y Herrnstein (1985) formulan que los niños con dificultades académicas, y con problemas al solucionar conflictos, presentan una "imagen desfavorable" ante sus pares y profesores; lo cual puede predisponer a su vez, a los profesores a interactuar diferencialmente con estos, a percibirlos como poco competentes en lo académico, lo 
que también puede llevar a los niños a considerar como poco gratificante el trabajo académico. Es probable que así, el contexto social del niño estimule el abandono de la escuela y otros cursos de acción que conducen a su posible inserción en un ambiente delictivo. Es decir, esas percepciones pueden constituir un factor disposicional que facilite o inhiba diferentes patrones de comportamiento, por lo tanto deben ser estudiados con detalle en el medio escolar (Mayer, 1995).

Desde la perspectiva de desarrollo, el estudio de la coerción ha recibido mucha atención dentro del área, por las consecuencias a corto y a largo plazo que se observan en las personas que la exhiben y en las que la reciben. Además de que este tipo de comportamiento afecta a los pares, los profesores, los familiares y los amigos en las relaciones cotidianas y puede exacerbarse con el paso del tiempo. Así, los estudios sobre coerción en niños y adolescentes indican que el patrón de comportamiento, en ocasiones, tiende a ser estable en el tiempo y agudizarse hasta convertirse en trasgresiones mayores (Cairns \& Cairns, 1994; Cairns, Santoyo \& Holly, 1994; Coie \& Dodge, 1998; Olweus, 1979). Otros estudios han encontrado que los niños agresivos muestran escasa conducta pro-social ${ }^{2}$ y pobre logro académico (Chen, Li \& Rubin, 1997). También se ha evidenciado que los niños agresivos tienen menos habilidades para relacionarse con sus pares y además presentan comportamiento académico bajo (French, 1988; Ladd \& Burgess, 1999).

Con respecto a la percepción de comportamientos coercitivos, hay poca concordancia entre las percepciones de los niños y el que terceras personas tienen sobre tales comportamientos (Boivin \& Bègin, 1989; Patterson, Kupersmidt \& Griesler, 1990). Dicho desacuerdo se acentúa en niños pequeños porque tienden a sobreestimar su competencia social en varios dominios. Sin embargo, aunque las percepciones de niños mayores son más precisas, hay niños que continúan con este sesgo, especialmente los niños coercitivos (Dodge \& Frame, 1982). Chang (2003) supone que las actitudes de los profesores hacia comportamientos coercitivos y aislados afecta la descripción que esos niños tienen de sí mismos. Así, el comportamiento y las actitudes de los demás tienen un sentido específico para cada persona en su propio contexto (Oñate, 1989). En general, los niños coercitivos tienden a sobreestimar su comportamiento social en comparación con la percepción que de ellos tienen sus profesores (Cillessen, van ljzendoorn, van Lieshout \& Hartup, 1992; Cairns \& Cairns, 1994).

Hallazgos sobre el efecto de la ecología social sugieren que el comportamiento coercitivo puede ser reforzado por los compañeros en algunas escue-

2. Aquél encaminado a satisfacer las metas institucionales del grupo (p.e., juego grupal y comportamiento académico) (López, 1984). 
las, es decir, los niños coercitivos pueden relacionarse con niños que apoyen ese comportamiento (Cairns, 1979) e incluso con aquellos ubicados en altas posiciones sociales (Estell, Cairns, Farmer y Cairns, 2002).

Uno de los primeros estudios realizados en México a través de observación directa en escenarios naturales, fue el desarrollado por Santoyo, Espinosa y Maciel (1996), quienes al utilizar el Sistema de Observación Conductual de las Interacciones Sociales (SOC-IS) obtuvieron datos sobre reciprocidad social positiva y negativa. En tal estudio los niños con comportamiento coercitivo muestran mayor frecuencia y duración de episodios negativos que ellos inician, que los niños no coercitivos del grupo de comparación. Por otro lado, los niños a los que se les dirige la coerción reciben más conducta negativa de la que ellos emiten y se involucran con mayor frecuencia en interacciones aversivas que otros inician (Maciel, 1996).

El estudio realizado por González (1998) también utilizó el SOC-IS, y mediante la estrategia de los Mapas Socioconductuales, reportó que los niños con comportamiento coercitivo pueden ser ubicados de diferentes formas en una estructura social: los nucleares; los rechazados y por consiguiente los que están aislados; y, finalmente, los niños que se asocian con otros niños con comportamiento coercitivo, constituyen grupos cohesivos. Sin embargo, en tales estudios no se utilizó una estrategia multi-método que permitiera analizar la posible interacción de diferentes niveles de análisis, y así una mayor comprensión del desarrollo integral de procesos coercitivos. Por ejemplo, el estudio de Rubio (2004) utilizó una estrategia multi-método, la cual permitió entender el comportamiento de niños con necesidades educativas especiales desde niveles de análisis complementarios; un aspecto relevante fue el uso del Inventario de Comportamientos Sociales y Académicos, ICSA (Rubio \& Santoyo, 2004), que se derivó del SOC-IS (Santoyo, Espinosa \& Bachá, 1994); lo que garantiza una mayor convergencia entre sus constructos teóricos. El ICSA contiene tres factores sustantivos y complementarios; en cada uno de ellos se desprenden categorías que agrupan a los reactivos en función de su contenido; tres para la interacción bajo conflicto (emisiones, recepciones y eventos negativos hacia pares y profesores), cuatro para áreas escolares, complementarias y de desempeño escolar y seis para las interacciones sociales, emisiones y recepciones hacia los pares y profesores. Aunque el estudio de Rubio (2004) no abordó procesos coercitivos mostró su utilidad como un estudio multi-método para el estudio de la organización del comportamiento in situ.

Los estudios que se realizan en escenarios naturales mediante el procedimiento de la metodología observacional constituyen una fuente de información importante para conocer una amplia gama de eventos interactivos, porque se identifica quiénes inician, mantienen y desarrollan las interacciones sociales en cuestión. Además, dichos estudios se derivan de la perspectiva 
de la síntesis de desarrollo, la cual supone que el comportamiento es un continuo de procesos dinámicos de reacciones entre la persona y el contexto social (Cairns, 1979). En suma, los estudios anteriores permiten reafirmar la postura conceptual y metodológica que se está proponiendo, y la pertinencia del enfoque multi-método para comprender la organización del comportamiento de niños escolares en Riesgo. El estudio de los conflictos diádicos desde la perspectiva de la síntesis de desarrollo, representa una propuesta teórica y metodológica, que ubica al proceso coercitivo en un continuo de tiempo y espacio (Magnuson \& Stattin, 1998). Dicha perspectiva proporciona un punto de vista comprensivo e integral del comportamiento coercitivo, porque supone que el comportamiento se organiza en subsistemas múltiples. Aunado a lo anterior, también se consideran eventos derivados de las interacciones escolares que pueden contribuir al desarrollo del comportamiento coercitivo (eventos disposicionales) porque cualquier cambio en la forma de interacción entre el ambiente y la persona involucrada puede afectar las relaciones subsecuentes (Bijou \& Baer, 1975). Es así como las interacciones coercitivas pueden incrementar la probabilidad de respuestas aversivas, tanto de quien las propicia como de quien las contesta, o incluso de quienes las observan sin ser partícipes directos (Mayer, 1995). De esta forma, es necesario comprender la organización del comportamiento, y no sólo el producto o el resultado aislado de un episodio de comportamiento entre individuos.

Por tal motivo, se plantea una propuesta de síntesis que implica a la metodología observacional, como una estrategia adecuada para evaluar la organización del comportamiento coercitivo in situ y en forma directa. A través de dicha estrategia se obtienen datos sobre aspectos dinámicos del comportamiento interactivo, de forma sistemática basados en patrones de comportamiento y no en variables aisladas (Cairns, Bergman \& Kagan, 1998; Magnusson, 1998). De esta forma, el propósito general de este trabajo es evaluar y extender la Generalidad del Modelo Wilson-Herrnstein a procesos de desarrollo. Y de forma específica, los propósitos son: a) Comparar la percepción del comportamiento social ante conflicto, social y académico entre niños Riesgo y Control acoplados, y entre la auto-descripción y la descripción del profesor; b) Examinar cómo la ecología social impacta en la organización del comportamiento coercitivo; c) Evaluar la organización y estabilidad del comportamiento de niños que exhiben comportamiento coercitivo; d) Identificar los eventos que desencadenan los intercambios coercitivos y los factores asociados. Para dichos fines, se compara la coherencia entre la auto-descripción y la percepción de los otros con respecto a comportamientos escolares. También se evalúan las relaciones sociales que promueven el comportamiento coercitivo de los niños Riesgo. Finalmente, se pretende evaluar, mediante un micro-análisis las propiedades secuenciales de los episodios negativos y del tiempo dedicado a la tarea académica. 


\section{MÉTODO}

\section{Participantes}

La muestra se conformó por 20 niños de tres grupos de tercer año, con sus tres profesores. La edad de los niños oscilaba entre 8 y 9 años de edad, 10 de ellos identificados en Riesgo de comportamiento coercitivo, de los cuales 8 fueron varones y 2 mujeres. Los niños se eligieron por tres criterios: nominación de tres adultos de forma independiente (Cairns \& Cairns, 1994), puntaje mayor a la media teórica de la escala de Comportamiento social ante conflicto (Rubio \& Santoyo, 2004), y consenso de los pares sobre conflictos recientes (Cairns \& Cairns, 1994). Y los diez niños restantes fueron Controles acoplados.

\section{Escenario}

La Dirección General de Servicios Educativos de la delegación política correspondiente autorizó la realización del estudio en una escuela primaria pública mixta, ubicada en el oriente de la Ciudad de México. Los datos se recolectaron en dos escenarios: en el aula, durante las sesiones de clase, y en el patio, durante el recreo. Cada salón contaba con un pizarrón, estantes, escritorio para profesor, mesas y sillas para dos niños en cada una de ellas.

\section{Aparatos y Mediciones}

Se utilizaron tablas, cronómetros, lápices, videocámara y hojas de registro como material de apoyo para recopilar la información del escenario escolar en los dos periodos de observación.

El Sistema de Observación Conductual de las Interacciones Sociales, SOC-IS (Santoyo et al, 1994). Detecta las actividades de los niños: identificando la dirección de los intercambios sociales, el lugar en donde ocurren, el nombre de los sujetos involucrados, la solución y los descriptores de cada episodio. Está compuesto por categorías excluyentes y exhaustivas, las cuales representan el comportamiento que los niños exhiben en el escenario escolar; comportamiento individual, académico y el comportamiento no académico, las actividades diádicas y de grupo o interacciones sociales; por ejemplo el comportamiento negativo, el juego en grupo y la negociación.

Las observaciones se recolectaron con la ayuda de dos parejas de observadores entrenados previamente para el uso del SOC-IS. Los observadores estuvieron en entrenamiento durante tres semanas, aproximadamente. Se consideró a un observador como capacitado, cuando mantuviera una concordancia del $80 \%$ con otro observador, durante cinco días consecutivos. El criterio de aceptación de los registros observacionales, obtenidos de forma independiente, fue que tuvieran una concordancia promedio entre observadores del $80 \%$. Posteriormente, para tener un mayor control de los registros 
se analizaron con la Kappa de Cohen, la cual corrige los acuerdos entre observadores debidos al azar. Los valores obtenidos para este estudio, fueron en promedio de .75 , lo que indica concordancia excelente de acuerdo con Bakeman y Gottman (1986). Las categorías empleadas del sistema de observación se definen en el Apéndice 1.

El Inventario de Comportamientos Sociales y Académicos, ICSA (Rubio \& Santoyo, 2004. Se derivó del SOC-IS (Santoyo et al, 1994) y fue diseñado para estimar el comportamiento social y académico de niños escolares a través de evaluaciones propias y de los adultos. El ICSA está conformado por 83 reactivos, tipo Likert pictográfico, de cinco puntos, repartidos en tres escalas. La consistencia interna obtenida para cada una de ellas fue alta. Se obtuvieron coeficientes Alpha de Cronbach con niveles aceptables para cada componente del inventario en las dos versiones, en la del profesor y en la de auto-descripción: Comportamiento Social con 41 reactivos y alfa $\alpha=.89$ y .77; Comportamiento Social ante Conflicto con 26 reactivos y valores $\alpha=.97$ y .92; y Comportamiento Académico con 16 reactivos y $\alpha=.89$ y .70 , respectivamente.

Entrevistas individuales. Conducidas en los tres grupos de tercer año (A, B y C) para evaluar los vínculos sociales, dicha estrategia se derivó del estudio de Cairns y Cairns (1994). Así, se les preguntó a los niños: "En tu salón de clases ¿quiénes se juntan mucho?", la respuesta era libre y podían nombrar a muchas personas o grupos como desearan. Si los niños no se llegaban a incluir en un subgrupo, se les preguntaba: "Y que hay de ti... ¿Hay un grupo con el que te juntas?". De dicha información se elaboraron Mapas Sociocognitivos Compuestos (MSCC) para conocer la estructura de las redes sociales de todo el salón y se eligieron sólo aquellas conexiones, que fueron estadísticamente significativas, como se especifica en la sección de procedimiento.

La entrevista se complementó con preguntas sobre conflictos recientes, las cuales se le hicieron a los mismos niños: "¿qué niños te molestan más?", "En las últimas semanas ¿hubo alguien que te molestara?, "Platícame sobre el último conflicto que hayas tenido en la escuela". Dicha estrategia garantizó que los niños respondieran sobre peleas y rumores cuando respondían negativamente a la primera pregunta. Las entrevistas fueron video grabadas y registradas en hojas para análisis posteriores.

Nominación del profesor sobre niños en Riesgo de comportamiento coercitivo de manera independiente. Debido al supuesto de que los profesores son los que mejor conocen a los niños en la escuela porque interactúan cotidianamente con ellos, pueden identificar su comportamiento en distintas áreas, además de distinguir a los niños con comportamiento coercitivo (Cairns \& Cairns, 1994; Santoyo \& Rubio, 2006). 
Procedimiento

Los niños focales se eligieron exclusivamente por los siguientes criterios:

Primero se entrevistó a los profesores de tercer año y se les preguntó sobre los niños más conflictivos en su salón de clases, la redacción de esa pregunta se modificó para poder planteárselas a los profesores del ciclo escolar anterior y al director de la escuela. El niño se identificó como Riesgo cuando, además de ser identificado como conflictivo por su profesor de tercer año, también fue identificado como tal por su profesor y/o por el director de la escuela. Segundo, a los profesores de tercer año se les proporcionó el ICSA para que respondiera el de cada niño sobre su comportamiento social ante conflicto. Los valores cercanos a cinco describen la característica de la persona evaluada, sólo se eligieron aquellos niños que según el instrumento casi siempre se involucran en situaciones de conflicto, esto es, con valores de 4 y 5 . Tercero, la entrevista sobre conflictos recientes se llevó a cabo en un salón de clases, de donde se obtuvieron frecuencias de los niños que fueron más nominados como conflictivos por consenso social, el criterio considerado es que fueran nombrados más del $50 \%$ de veces por sus pares. Después de identificar a los niños Riesgo, se acoplaron 10 niños como grupo de Comparación, con cada uno de los primeros, en edad, sexo y grado escolar. Los datos se recolectaron en dos cortes anuales, cuando los participantes estaban en tercero y cuando pasaron a cuarto grado. ${ }^{3}$

Por otra parte, se aplicó colectivamente a cada grupo de tercer grado, la versión de auto-descripción del ICSA, la cual respondieron individualmente en el salón de clases con una duración aproximada de 45 minutos por grupo. Las respuestas proporcionadas en la entrevista individual sobre vínculos sociales, sirvieron para diseñar los MSCC. Primero, en una matriz de doble entrada se ubican a los entrevistados y a los nominados; en las filas se ubican las personas que nomina cada niño entrevistado (columnas). Esta matriz de recuerdo (Farmer \& Cairns, 1991) se llena con una letra que indica el grupo o grupos de membresía. Segundo, a partir de los datos de la matriz de recuerdo se hace otra matriz que se llama de coocurrencia, la cual contiene el número de veces que pares de niños son nombrados en un grupo por consenso social. Tercero, cada columna de la matriz de coocurrencia se correlacionó con todas las demás. La identificación de conexiones significativas se determinó mediante el criterio de decisión: si el patrón de asociación de cada niño tenía una correlación superior a .40 , con probabilidad de error menor a .01. Este procedimiento no se limitó a que los niños respondieran sólo a su círculo de amigos, sino a todos los que se encontraban en su grupo. A partir de esa información se determinó el peso de sus conexiones, considerando

3. En el segundo corte a los niños se les asignó diferente profesor. 
tres clases de relaciones significativas con base a sus desviaciones estándar $(D E)$ sobre la media global: Débiles $(+1 D E)$; Moderadas (+1.5 $D E)$; Fuertes (+2 DE).

Finalmente, los niños seleccionados de tercer año, Riesgo y Controles acoplados, fueron observados diariamente con el SOC-IS en situaciones naturales de acuerdo con las actividades cotidianas que programaba la institución. Las observaciones las realizaban parejas de observadores previamente entrenados. Cada pareja de observadores registraba a los niños en el recreo y en el salón de clases durante 10 y 15 minutos respectivamente, por cinco sesiones consecutivas en cada escenario. Para mayor control de los datos observacionales, los observadores desconocían el estatus de los niños observados para evitar prejuicios sobre el comportamiento de los participantes.

Los datos de observación directa y de evaluación indirecta se recolectaron a la mitad del ciclo escolar, cuando los niños estaban en tercer grado y cuando pasaron a cuarto grado. El orden que se describe en el procedimiento es el mismo en el que se obtuvo la información.

\section{RESULTADOS}

Con la finalidad de evaluar los efectos principales de grupo (Riesgo o Control) y corte (1 y 2), así como los efectos de interacción entre factores se decidió realizar un análisis de varianza mixto. Para el análisis secuencial se procedió a realizar un análisis de contingencia no paramétrico.

Percepción del comportamiento social y académico de los niños.

En primer lugar se muestran los datos que provienen del inventario de comportamientos sociales y académicos desde las dos perspectivas, niño y profesor. La Figura 1 muestra la auto-descripción y la descripción del profesor sobre tres categorías del comportamiento social ante conflicto (EN= Emisión Negativa, RN= Recepción Negativa y $\mathrm{SN}=$ Social Negativa) en dos cortes de observación. Los niños Riesgo y de Comparación se auto-describen como que casi nunca, emiten, reciben o se involucran en situaciones de conflicto en los dos cortes de observación. No hay diferencias estadísticamente significativas, entre corte, ni entre grupos.

Para la perspectiva del profesor se realizó un análisis de varianza (ANOVA) de dos factores (corte de observación y estatus del niño) con medidas repetidas en el corte de observación. El análisis muestra diferencias estadísticamente significativas entre grupos, el profesor describe que los niños Riesgo emiten $F(1,18)=17.345, p=001$, reciben $F(1,18)=26.800, p=.001 \mathrm{y}$ se involucran más en episodios negativos $F(1,18)=15.917, p=.001$ que los niños Control, en el primer corte de observación. 
En el segundo corte, la percepción que el profesor tenía de ambos grupos se modificó, igualando la descripción que hace de ambos en las categorías emisión $F(1,18)=17.591, p=.01$ y social negativo $F(1,18)=18.051, p=.001$. Además, el efecto principal del corte de observación $F(1,18)=5.404, p=.03$ en la categoría social negativo, indica que independientemente del estatus del niño, el profesor percibe diferencias de un corte de observación a otro.

Descripción del Comportamiento Social ante Conflicto

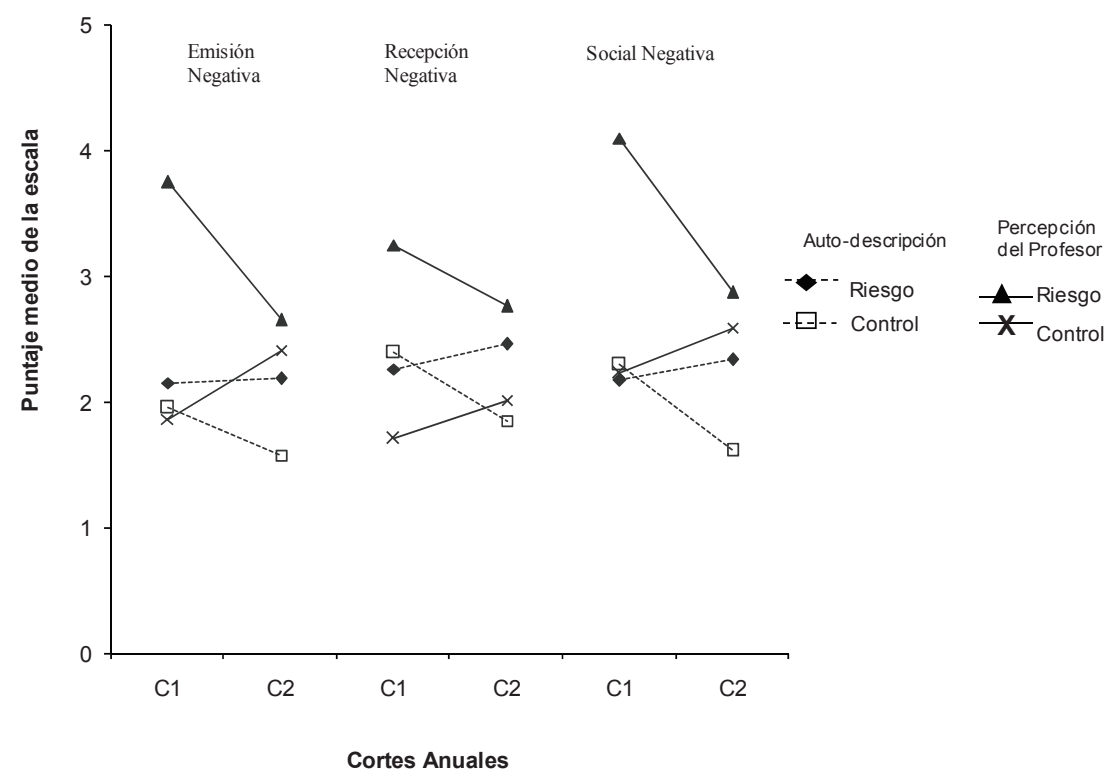

Figura 1. Puntajes promedio de las categorías del Comportamiento Social ante Conflicto ( $E N=$ Emisión Negativa, $R N=$ Recepción Negativa $Y S N=$ Social Negativa) desde las dos perspectivas (profesor y niño) en dos periodos de observación (C1= corte uno y C2= corte dos). Valores cercanos a cinco describe más la característica de la persona evaluada, y valores cercanos a uno describe menos la característica de la persona evaluada.

En la Figura 2 se presentan los datos de auto-descripción y descripción del profesor sobre las dimensiones del comportamiento académico $(\mathrm{MB}=$ Materias Básicas, $\mathrm{MC}=$ Materias Complementarias, $\mathrm{D}=$ Distracción y $\mathrm{P}=$ Persisten- 
cia). No se observan diferencias en la auto-descripción que hacen los niños Riesgo y Control sobre las categorías del comportamiento académico, en ninguno de los cortes de observación.

Se realizó un ANOVA mixto para la versión del profesor, el cual indica que hay un efecto estadísticamente significativo entre grupos, es decir, el profesor describe a los niños Control con puntajes altos en las materias básicas $F(1,18)=12.285, p=.003$, complementarias $F(1,18)=6.672, p<=.01$ y en persistencia de la tarea $F(1,18)=8.246, p=.01$, en los dos cortes de observación. El efecto principal del corte de observación $F(1,18)=5.324, p=$ .03 , en la categoría materias complementarias, indica que el profesor les da puntajes más altos a ambos grupos en el segundo corte de observación en comparación al que les da en el primero.

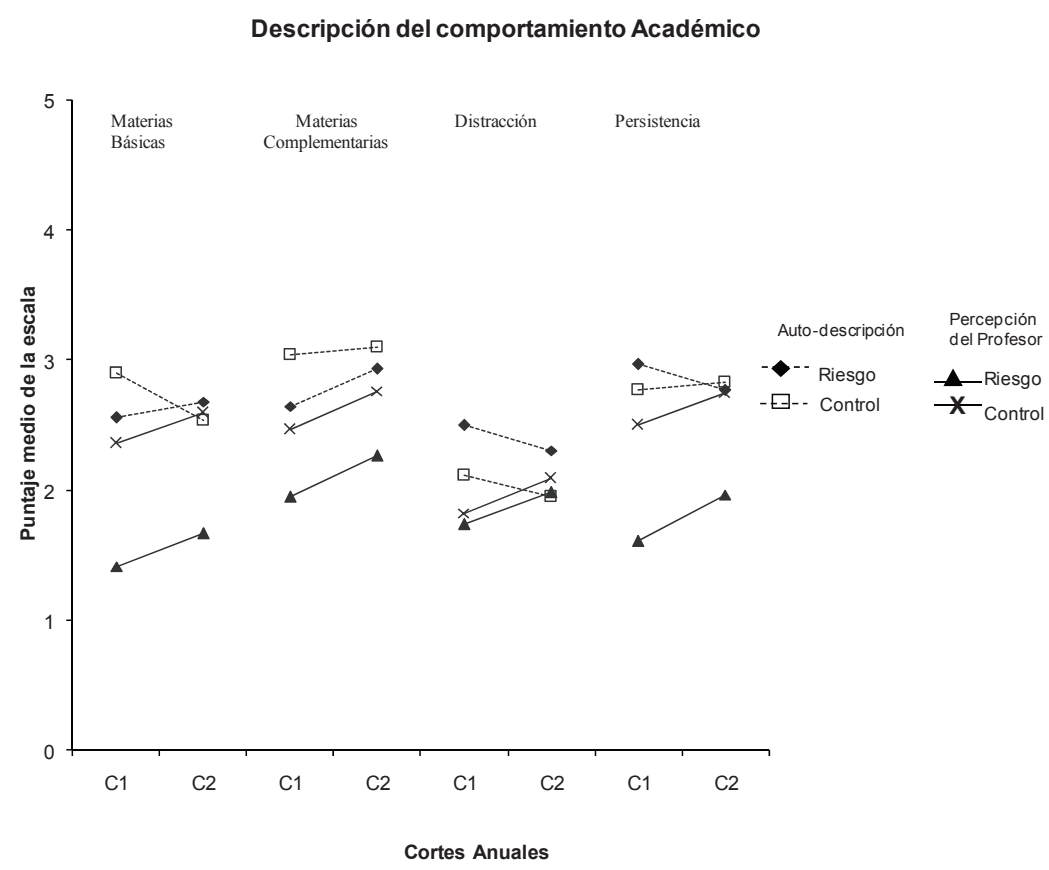

Figura 2. Puntajes promedio de las categorías del Comportamiento Académico (MB=Materias Básicas, $M C=$ Materias Complementarias, $D=$ Distracción y $P=$ Persistencia) desde las dos perspectivas en dos periodos de observación (C1= corte uno y C2= corte dos). Valores cercanos a cinco describe más la característica de la persona evaluada, y valores cercanos a uno describe menos la característica de la persona evaluada. 
En el Factor de Comportamiento Social, la auto-descripción de los niños Riesgo y Control sobre las dimensiones del comportamiento social (Correspondencia, Efectividad y Reciprocidad Social; Emisiones, Recepciones y Episodios Sociales) es similar a la descripción que hace el profesor de éstos en los dos cortes de observación. No encontrándose diferencias estadísticamente significativas por corte o grupo (ver Tabla 1).

Puntaje promedio de las Categorías de Auto-descripción del Comportamiento Social

\begin{tabular}{lcccc}
\hline Categorías & \multicolumn{2}{c}{ riesgo } & \multicolumn{2}{c}{ control } \\
\cline { 2 - 5 } & C1 & C2 & C1 & C2 \\
\hline Correspondencia Social & 3.35 & 3.46 & 3.80 & 3.56 \\
Efectividad Social & 3.56 & 3.41 & 3.33 & 4.18 \\
Recepciones & 3.42 & 3.97 & 3.57 & 3.65 \\
Reciprocidad Social & 3.76 & 3.38 & 3.45 & 3.68 \\
Sociales & 3.05 & 2.68 & 2.85 & 2.89 \\
Emisiones & 3.17 & 3.70 & 2.95 & 3.49 \\
\hline
\end{tabular}

Puntaje promedio de las Categorías de Descripción del profesor sobre el Comportamiento Social

\begin{tabular}{lcccc}
\hline Categorías & \multicolumn{2}{c}{ riesgo } & \multicolumn{2}{c}{ control } \\
\cline { 2 - 5 } & C1 & C2 & C1 & C2 \\
\hline Correspondencia Social & 4.14 & 3.90 & 3.71 & 4.18 \\
Efectividad Social & 3.86 & 3.63 & 3.51 & 3.86 \\
Recepciones & 3.64 & 4.30 & 3.88 & 4.42 \\
Reciprocidad Social & 3.64 & 3.70 & 3.88 & 4.18 \\
Sociales & 2.91 & 3.14 & 3.05 & 3.37 \\
Emisiones & 3.82 & 4.22 & 3.60 & 4.62 \\
\hline
\end{tabular}

Tabla 1. Puntaje Promedio de las categorías del Comportamiento Social (CS= Correspondencia Social, ES=Efectividad Social, $R=$ Recepciones, $R S=R e c i p r o c i d a d$ Social, $S=$ Social y $E=E$ Emisiones desde las dos perspectivas en dos cortes de observación (C1= corte uno y C2= corte dos).

Mapas Sociales de los niños

En relación a los MSCC de los tres grupos de tercer grado, se identifican subgrupos de niños y/o niñas, con pocas relaciones y con muchas relaciones. 
Los niños Riesgo, al igual que los niños Control se relacionan de diferentes formas en la red social de su grupo, esto es, se identifican niños Riesgo que se relacionan con niños Riesgo y con niños que no están en riesgo. En la Tabla 2 se presenta el número de veces que dicen 'los niños' que se 'juntan'. En el primer corte de observación se observan diferencias entre los niños Riesgo y Control porque los primeros tienen mayor número de conexiones (63). Otro dato importante es que cuatro niños Riesgo Mr, FEA, Fr, y JA tienen conexiones fuertes entre ellos mismos. La parte inferior de la Tabla 2 muestra el segundo corte e indica que las diferencias observadas entre los niños Riesgo y Control son en otro sentido, ahora los primeros tienen menor número de conexiones con sus pares (58). Es importante notar que el número total de conexiones de los niños Control se duplica en el segundo corte respecto al primero. Además las cuatro conexiones fuertes que se habían identificado entre niños Riesgo cambiaron, ahora sólo se identifican dos conexiones moderadas entre ellos. En cambio, los niños Control tienen conexiones fuertes, moderadas y débiles con los niños Riesgo.

Conexiones totales ponderadas de los niños focales en dos cortes de observación

\begin{tabular}{cccccc}
\hline \multicolumn{6}{c}{ Primer corte de observación. } \\
\hline Riesgo & $\Sigma$ & $\begin{array}{c}\text { Conexiones con } \\
\text { coercitivos }\end{array}$ & Control & $\Sigma$ & $\begin{array}{c}\text { Conexiones con } \\
\text { coercitivos }\end{array}$ \\
VB & 3 & 0 & LA & 1.5 & 0 \\
LD & 0 & 0 & VE & 12.5 & 0 \\
D & 10 & $4 \mathrm{f}$ & Om & 3.5 & 0 \\
Mr & 10 & $4 \mathrm{f}$ & $\mathrm{S}$ & 0 & 0 \\
FEA & 9.5 & $4 \mathrm{f}$ & $\mathrm{L}$ & 4 & 0 \\
Fr & 10 & $4 \mathrm{f}$ & $\mathrm{JE}$ & 0 & 0 \\
JA & 10 & $4 \mathrm{f}$ & $\mathrm{J}$ & 9.5 & 0 \\
M & 2 & 0 & Os & 1.5 & 0 \\
D & 5.5 & 0 & $\mathrm{Mi}$ & 9 & 0 \\
BD & 3 & 0 & $\mathrm{~F}$ & 4 & 0 \\
\hline$\Sigma$ & 63 & $20 \mathrm{f}$ & $\Sigma$ & 45.5 & 0 \\
\hline
\end{tabular}

$\mathrm{f}=$ fuertes, $\mathrm{m}=$ moderadas, $\mathrm{y} d=$ débiles 


\begin{tabular}{cccccc}
\hline \multicolumn{5}{c}{ Segundo corte de observación. } \\
\hline Riesgo & $\Sigma$ & $\begin{array}{c}\text { Conexiones con } \\
\text { coercitivos }\end{array}$ & Control & $\Sigma$ & $\begin{array}{c}\text { Conexiones con } \\
\text { coercitivos }\end{array}$ \\
VB & 14 & 0 & LA & 5.5 & 0 \\
LD & 0 & 0 & VE & 16 & 0 \\
D & 3.5 & 0 & Om & 8 & 0 \\
Mr & 8.5 & $1 \mathrm{~m}$ & $\mathrm{~S}$ & 4.5 & $1 \mathrm{~m}$ \\
FEA & 9.5 & 0 & $\mathrm{~L}$ & 13.5 & $1 \mathrm{~m}$ \\
Fr & 6.5 & $1 \mathrm{~m}$ & JE & 5 & $1 \mathrm{~m}$ \\
JA & 4.5 & 0 & $\mathrm{~J}$ & 8 & $1 \mathrm{~d}$ \\
M & 3.5 & 0 & Os & 3.5 & 0 \\
D & 4 & 0 & Mi & 13.5 & $1 \mathrm{~m}$ \\
BD & 4 & 0 & $\mathrm{~F}$ & 14 & $1 \mathrm{f}$ \\
\hline$\Sigma$ & 58 & $2 \mathrm{~m}$ & $\sum$ & 91.5 & $1 \mathrm{f}, 4 \mathrm{~m}, 1 \mathrm{~d}$ \\
\hline
\end{tabular}

$\mathrm{f}=$ fuertes, $\mathrm{m}=$ moderadas, $\mathrm{y} d=$ débiles

Tabla 2. Relaciones ponderadas de los niños Riesgo y Control de tercer grado en dos periodos de observación (C1= corte uno y $C 2=$ corte dos).

Organización del Comportamiento Social y Académico de los niños.

En la Tabla 3 se sintetiza la frecuencia y el tipo de conflicto en el que se involucran los niños Riesgo y Control en el aula y recreo. Esto es, la información que se presenta en la tabla se derivó del contenido de las interacciones negativas de los niños. Es importante resaltar que los niños Riesgo se involucran con mayor frecuencia en episodios negativos de tipo físico, que son provocados por sus pares, en el corte uno; pero en el segundo periodo de observación, la coerción verbal provocada por los pares, aumenta y disminuye la física. Además, los niños Riesgo reciben más regaños del profesor que los niños del grupo Control, en ambos periodos. 
Categorías conductuales de los episodios negativos derivados del Sistema Conductual de las Interacciones Sociales, SOC-IS

\begin{tabular}{|c|c|c|c|c|c|}
\hline \multirow[t]{2}{*}{ Categoría } & \multirow[t]{2}{*}{ Ejemplos } & \multicolumn{2}{|c|}{$\begin{array}{c}\text { FR Epn } \\
\text { Riesgo }\end{array}$} & \multicolumn{2}{|c|}{$\begin{array}{l}\text { FR Epn } \\
\text { Control }\end{array}$} \\
\hline & & C1 & C2 & C1 & C2 \\
\hline Coerción física & $\begin{array}{c}\text { Empujar, Pegar, Dar manotazos, } \\
\text { Ahorcar, Atacar a alguien, } \\
\text { Provocar una pelea, Dar una } \\
\text { paliza. }\end{array}$ & 14 & 8 & 2 & 0 \\
\hline Coerción Verbal & $\begin{array}{c}\text { Insultos Verbales, Amenazas } \\
\text { hostiles, Humillación verbal, } \\
\text { Gritarle a alguien, Maldecir, } \\
\text { Discutir, Molestar, Insultar, Burlas } \\
\text { Hostiles y Criticar. }\end{array}$ & 2 & 11 & 0 & 2 \\
\hline $\begin{array}{l}\text { Regaños del } \\
\text { Profesor }\end{array}$ & $\begin{array}{l}\text { Castigar y poner en evidencia } \\
\text { al niño delante del grupo por } \\
\text { percepción de mal comportamiento }\end{array}$ & 23 & 15 & 4 & 2 \\
\hline$\Sigma$ & & 39 & 34 & 6 & 4 \\
\hline
\end{tabular}

FR Epn= Frecuencia de episodios negativos.

$\mathrm{C} 1=$ corte uno $\mathrm{C} 2$ = corte dos

Tabla 3. Frecuencia, Tipos de Conflictos y Contenido de episodios negativos derivados del Sistema de Observación Conductual de las Interacciones Sociales, SOC-IS en dos periodos de observación (C1= corte uno y C2= corte dos).

En la Figura 3 se incluye la distribución del tiempo dedicado a la actividad académica en relación al ocio. En la parte superior de la Figura 3 se observa que en el corte uno algunos niños Riesgo (3) y Control (4) le dedican mayor tiempo a la actividad académicas instruida por el profesor que a otras respuestas. En la parte inferior de la Figura 3 se observan diferencias estadísticamente significativas de un corte de observación a otro $F(1,18)=5.948, p=$ .05 porque en el segundo corte los niños Riesgo y Control le dedican mayor tiempo a otras respuestas que a las actividades académicas. 
Duración Relativa de Actividad Académica y Otras Respuestas de los niños riesgo.

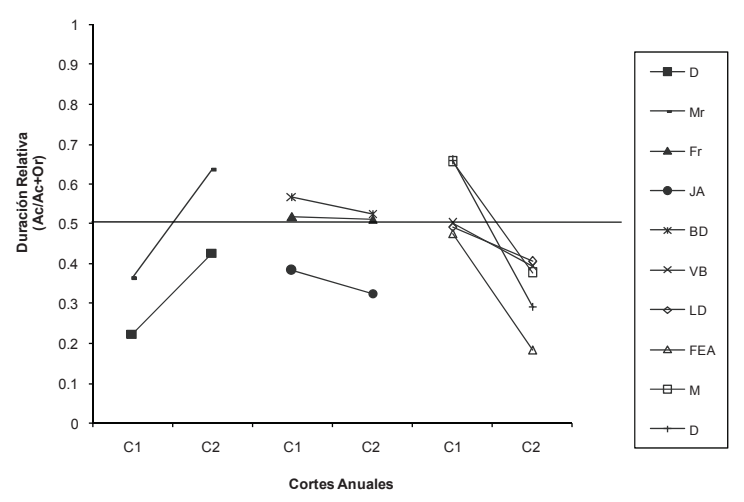

Duración Relativa de Actividad Académica y Otras Respuestas de los niños control.

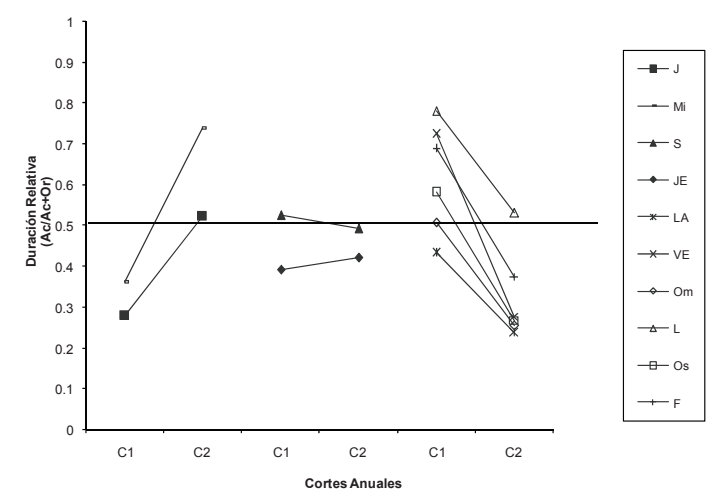

$\mathrm{Ac}=$ Actividad Académica $\mathrm{Or}=$ Ocio

$\mathrm{C} 1=$ corte uno $\mathrm{C} 2=$ corte dos

Datos cercanos a uno indica que los niños focales le dedican mayor tiempo a las actividades académicas instruidas por el profesor. En cambio, valores menores a .5 indican que los niños focales le dedican mayor tiempo al ocio (mirar hacia un punto y/o quedarse callado).

Figura 3. Tiempo relativo asignado a actividades académicas y al ocio en el aula, en dos periodos de observación (C1 y C2, cortes uno y dos). Datos cercanos a 1 implican mayor dedicación a la actividad académica, mientras que datos próximos a 0 implican mayor dedicación a ocio. Los datos del lado izquierdo y derecho de la gráfica muestran trayectorias ascendentes y descendentes, respectivamente. Al centro se representan los participantes con trayectorias estables. 
Estímulos controladores de episodios coercitivos.

Se incluye el análisis secuencial para evaluar si después de una provocación (emisiones y recepciones negativas) los niños Riesgo y de Comparación se involucran en episodios negativos (respuesta a esas provocaciones). EI micro-análisis de inicios y episodios negativos muestra la frecuencia en la que los niños Riesgo y Control se involucran en episodios negativos dada una provocación, la probabilidad condicionada y la relación significativa entre categorías para cada grupo de niños.

La Tabla 4 muestra que los niños Riesgo si se involucran en episodios negativos dada una provocación en los dos cortes de observación, a diferencia de los niños Control, los cuales tienen episodios sociales positivos después de la provocación. La probabilidad condicional de que los niños Riesgo se involucren en episodios sociales negativos dada una provocación es alta para el primer corte de observación, además las relaciones significativas a partir de los residuos ajustados superiores a +1.96 , indican que las provocaciones son estímulos que facilitan los episodios negativos, y no son debidos al azar, mientras que las provocaciones para los niños Control son estímulos que inhiben episodios negativos $x^{2}(4, N=10)=8.347, p=.003$. En el corte dos, aunque la frecuencia de episodios negativos de los niños Riesgo disminuye, los residuos ajustados del análisis secuencial indican, igual que en el primer corte, que los episodios negativos son consecuencia de provocaciones que ellos reciben $x^{2}(4, N=10)=7.444, p=.01$.

Primer corte de observación

\begin{tabular}{cccc}
\hline Provocaciones & Episodios & $\begin{array}{c}\text { Episodios } \\
\text { Sogativos }\end{array}$ & 12 \\
\hline \multirow{7}{*}{ Riesgo } & FR & 25 & .32 \\
& PC & .68 & -2.9 \\
& RA & 2.9 & 5 \\
& FR & 0 & 1 \\
\hline & PC & 0 & 2.9 \\
\cline { 2 - 4 } & RA & -2.9 & \\
\hline
\end{tabular}


Segundo corte de observación

\begin{tabular}{cccc}
\hline Provocaciones & & $\begin{array}{c}\text { Episodios } \\
\text { Negativos }\end{array}$ & $\begin{array}{c}\text { Episodios } \\
\text { Sociales }\end{array}$ \\
\hline \multirow{6}{*}{ Riesgo } & FR & 15 & 4 \\
& PC & .79 & .21 \\
& RA & 2.7 & -2.7 \\
& FR & 0 & 5 \\
& PC & 0 & 1 \\
\cline { 2 - 4 } & RA & -2.9 & 2.9 \\
\hline
\end{tabular}

Provocaciones $=$ Emisión y/o recepción negativa

Frecuencia $=$ FR, Probabilidad Condicional $=$ PC, Residuos ajustados $=$ RA

Tabla 4. Patrón de comportamiento social negativo de los niños Riesgo y Control en dos periodos de observación (Frecuencia; Probabilidad Condicional; Residuos Ajustados).

En la Tabla 5 se muestra el análisis secuencial del tiempo que los niños Riesgo y Control le dedican a la actividad académica, como conductas antecedentes y los inicios que el profesor les dirige a ambos grupos, como consecuente. Los datos indican que los profesores les dirigen más inicios negativos (recepciones negativas) a los niños Riesgo cuando estos están en la actividad académica, con una probabilidad condicional de .36 en el primer corte de observación. Las relaciones significativas de los residuos ajustados superiores a +1.96 demuestra que hay una relación en las recepciones negativas que los profesores le dirigen a los niños Riesgo cuando están en actividad académica. En los niños Control, la frecuencia de ocurrencia entre esas conductas es menor, al igual que la probabilidad condicional $x^{2}(4, N=10)=9.436, p=$ .001. En el corte dos, el análisis de los residuos ajustados revela lo mismo que en el corte uno, el profesor les dirige más inicios negativos (recepciones negativas) que inicios positivos (recepciones positivas) a los niños Riesgo que a los niños de Control $x^{2}(4, N=10)=3.840, p=.05$ ) cuando ambos se encuentran en actividad académica. 
Primer corte de observación

\begin{tabular}{|c|c|c|c|}
\hline $\begin{array}{l}\text { Actividad } \\
\text { Académica }\end{array}$ & & $\begin{array}{c}\text { Inicios } \\
\text { Negativos }\end{array}$ & $\begin{array}{c}\text { Inicios } \\
\text { Positivos }\end{array}$ \\
\hline & FR & 49 & 87 \\
\hline \multirow[t]{3}{*}{ Riesgo } & PC & .36 & .64 \\
\hline & RA & 3.1 & -3.1 \\
\hline & FR & 12 & 63 \\
\hline \multirow[t]{2}{*}{ Control } & PC & .16 & .84 \\
\hline & RA & -3.1 & 3.1 \\
\hline \multicolumn{4}{|c|}{ Segundo corte de observación } \\
\hline \multirow[t]{2}{*}{$\begin{array}{c}\text { Actividad } \\
\text { Académica }\end{array}$} & & $\begin{array}{c}\text { Inicios } \\
\text { Negativos }\end{array}$ & $\begin{array}{c}\text { Inicios } \\
\text { Positivos }\end{array}$ \\
\hline & $\mathrm{FR}$ & 42 & 67 \\
\hline \multirow[t]{3}{*}{ Riesgo } & PC & .38 & .62 \\
\hline & RA & 2.0 & -2.0 \\
\hline & $\mathrm{FR}$ & 27 & 77 \\
\hline \multirow[t]{2}{*}{ Control } & PC & .26 & .74 \\
\hline & RA & -2.0 & 2.0 \\
\hline
\end{tabular}

Actividad Académica $=$ Tiempo dedicado a la actividad académica.

Frecuencia $=$ FR, Probabilidad Condicional $=$ PC, Residuos ajustados $=R A$

Tabla 5. Patrón de comportamiento académico de los niños Riesgo y Control en dos periodos de observación (Frecuencia; Probabilidad Condicional; Residuos Ajustados).

\section{DISCUSIÓN}

La postura conceptual y la estrategia metodológica de la investigación representan en sí mismas, contribuciones importantes en el campo de la psicología del desarrollo. En el aspecto teórico se confirma la conveniencia del modelo conductual y de interacción social (Cairns, 1979; Patterson, 1982; Wilson \& Herrnstein, 1985) para estudiar la organización del comportamiento coercitivo de niños escolares en Riesgo, porque se presenta evidencia de algunos factores de Riesgo que pueden constituir patrones de comportamiento coercitivo, por ejemplo: niños coercitivos que se asocian con pares que apoyan su comportamiento coercitivo (Cairns, 1979), escaso o nulo reconocimiento por actividades pro-sociales, en relación al que reciben por su comportamiento coercitivo (Wilson \& Herrnstein, 1985), el reforzamiento negativo que se deriva de su relación con los profesores cuando éstos tratan de disciplinarlos 
(Sidman, 1989). Además, los niños Riesgo en el primer corte son receptores frecuentes de actos coercitivos de los profesores aun y cuando se encuentren participando en actividad académica o actos pro-sociales, a diferencia de los niños Control, como lo indican los análisis de contingencia realizados en este estudio.

En términos de percepción, en el inventario de comportamientos sociales y académicos (ICSA) se encuentra que los niños subestiman su comportamiento social ante conflicto, es decir, no se representan como conflictivos. En cambio, la percepción del profesor distingue adecuadamente a los niños Control de los niños Riesgo, el hallazgo es consistente con estudios previos en los que se reporta que estos niños no necesariamente se describen como conflictivos en situaciones escolares (González, 1998). En los comportamientos "deseables" los niños Riesgo y Control se perciben de la misma forma que los percibe el profesor en comportamientos sociales, esto es, perciben que son aceptados por los pares (Cairns, Cairns, Neckerman, Gest \& Gariépy, 1988; Estell, et al, 2002). En comportamientos académicos, el profesor les asigna a los niños Riesgo un bajo comportamiento académico, esto coincide con lo reportado por Chen, Li y Rubin (1997) sobre la asociación negativa que hace el profesor sobre el comportamiento académico y social ante conflicto de los niños Riesgo. Estos hallazgos sugieren que el comportamiento auto-descriptivo es un subproducto de la socialización del niño y puede ser el reflejo de procesos interactivos (Santoyo \& Rubio, 2006), es decir, las creencias y el comportamiento del profesor ejercen influencia directa como eventos disposicionales dentro del escenario. Por ejemplo, el profesor percibe que en situaciones de conflicto, los niños Riesgo provocan los episodios negativos, a diferencia de los datos conductuales, los cuales muestran que dichos niños, más bien, reciben las provocaciones de sus compañeros.

Con respecto a las relaciones sociales, se confirma que los niños Riesgo al igual que los Control se ubican de diferentes formas en una estructura social, pueden relacionarse sólo con niños Riesgo, con muchos niños o estar aislados (González, 1998). Estos hallazgos muestran que el contexto de algunos niños puede no favorecer su integración al grupo, como en el caso de los niños Riesgo que se relacionan entre sí o que se mantienen aislados; pero también indican que para quienes mantienen relaciones con niños que exhiben comportamiento pro-social su aceptación dentro del grupo es más probable e incluso puede observarse una disminución en la participación de nuevos conflictos.

Por otra parte, las redes sociales son susceptibles de los efectos de desarrollo porque ciertas propiedades como la homogeneidad, expansión de la red y las conexiones significativas se van modificando constantemente (Santoyo, Colmenares \& Losada, 2007). En particular, en este estudio se identifica el proceso de expansión de la red social, pero sólo para el grupo Control, 
como puede verificarse en el segundo corte (en la Tabla 2). Así, es probable que el cambio en la configuración de la red social (nuevos compañeros, profesores y por tanto contingencias sociales) muestre una contribución diferencial en los niños de acuerdo con su historia de interacción y su estatus como Riesgo y Control. Este hallazgo tiene que ser más documentado en investigaciones futuras para identificar los mecanismos responsables de la expansión diferencial de la red social.

Sobre este punto, el que los niños cambien de grado escolar, de profesor y de compañeros, puede disminuir la probabilidad de que se desencadenen episodios sociales negativos porque en algunos salones y grupos sociales, la coerción es castigada y mal vista por los pares y profesores (Patterson, 1974), como ocurrió en este estudio, cuando se evaluó el segundo corte de observación. Es decir, la estabilidad en las afiliaciones del grupo es una función de la estabilidad de la composición del salón de clases, y tiene impacto en la estabilidad de los patrones de comportamiento coercitivo (Neckerman, 1996).

En el análisis sobre la organización del comportamiento, se encuentra que algunos niños Riesgo son aceptados por sus pares, tienen muchos intercambios sociales con los compañeros del salón, al igual que sus compañeros. En el aspecto académico es importante mencionar que tanto los niños Riesgo como los niños Control dedican tiempo equivalente a las actividades que el profesor les asigna, dichas similitudes ofrecen otra perspectiva que no coincide con las diferencias percibidas por el profesor sobre el factor académico. Este aspecto, también permite comprender la influencia del profesor sobre el comportamiento académico del niño. El que los niños Riesgo y Control le dediquen poco tiempo a la actividad académica, indica que los profesores no están siendo efectivos en las alternativas pro-sociales que les proporciona a los niños porque éstos no logran involucrarse en ella. Por ejemplo, en el primer corte de observación, el profesor se dirige con mayor frecuencia de forma coercitiva a los niños Riesgo, en comparación a los niños Control. Es posible que los eventos aversivos emitidos por el profesor, como eventos disposicionales o facilitadores, incrementen la probabilidad subsecuente de respuestas coercitivas en los niños Riesgo (Mayer, 1995; Patterson, 1974).

Sobre las interacciones sociales coercitivas, los resultados sobre frecuencia y dirección revelan diferencias entre los niños Riesgo y Control en situaciones de conflicto, los primeros exhiben actos aversivos en tasas más altas y con mayor frecuencia que los otros niños. Además, los episodios coercitivos en los que se involucran los niños Riesgo ocurren inmediatamente después de un inicio negativo previo, lo cual constituye un predictor de episodios coercitivos futuros (Maciel, 1996), y sugiere la operación del reforzamiento negativo como el mecanismo de control, en particular para los niños Riesgo de esta muestra, lo cual es consistente con la propuesta de Sidman (1989). 
El comportamiento coercitivo se forma y se mantiene por su valor funcional al tratar de suprimir respuestas aversiva de las personas que tratan de controlar el comportamiento de esa persona (Snyder \& Patterson, 1995). Es decir, se fortalece en la medida que se utiliza como escape en un ambiente aversivo, de esta forma los niños Riesgo al tener mayor reforzamiento negativo, en comparación con sus compañeros, reflejan su adaptación a las demandas que se les presentan en su ambiente escolar, lo cual puede predisponer este tipo de interacciones en el futuro con otras personas y en otros escenarios (Sidman, 1989).

Estos hallazgos coinciden con un supuesto importante de la síntesis de desarrollo que habla sobre la plasticidad del comportamiento, el cual puede adaptarse, ya sea para reorganizarse o para mantenerse a través del tiempo (Cairns, 1979). Los datos indican que el patrón de comportamiento coercitivo está en función de factores proximales de interacción que favorecen su desarrollo; el trato del profesor, las relaciones con pares similares en el comportamiento coercitivo, el ambiente coercitivo, la inconsistencia en las reglas y la aplicación de consecuencias. Esto es apoyado por el modelo de Wilson y Herrnstein (1985), el cual supone que la conducta es un proceso de elección, que será moldeado por una suma de factores. En esta muestra, el escenario escolar proporciona en el primer corte, suficientes oportunidades para fortalecer el desarrollo del comportamiento coercitivo, el trato diferenciado que reciben los niños Riesgo del profesor, que reciban más atención por su comportamiento coercitivo en comparación al que reciben por su comportamiento pro-social, la cohesión de la red de niños coercitivos, la percepción del profesor como conflictivos y con bajo comportamiento académico. De esta forma, el niño percibe como más gratificante el comportamiento coercitivo porque los beneficios que obtiene por este son inmediatos, como la atención que recibe de los profesores y la aprobación de sus pares. Finalmente, el reforzamiento relativo que el comportamiento pro-social recibe deberá incrementarse, lo que en consecuencia produciría un fortalecimiento relativo para estas acciones y, en consecuencia, un debilitamiento para las opciones coercitivas como lo propone el modelo de TNHEC (Wilson \& Herrnstein, 1985).

Por estas razones, se considera a la escuela como un determinante importante en la organización del comportamiento coercitivo, porque el ambiente coercitivo y punitivo que aparece en el salón de clases, es similar al que se ha identificado, en otros escenarios y estudios (Patterson, 1982).

Esto es, las interacciones cotidianas que se realizan en la escuela pueden consolidar patrones de comportamiento coercitivo en los niños Riesgo, por las oportunidades que perciben, y por los beneficios inmediatos que obtienen. Además, de que no se promueve eficientemente otro tipo de comportamiento, como el social, o el académico (Wilson \& Herrnstein, 1985). En realidad, el tiempo que ambas muestras dedican a la actividad académica 
respecto al ocio es muy pobre (menor al $40 \%$ ) y las trayectorias que los niños de ambas muestras exhiben es preocupante puesto que la mayoría tiende a disminuir el tiempo asignado relativo a actividad académica en el segundo corte. Del dato se concluye que la escuela no esta cumpliendo con las expectativas que se tienen de ella, promover en los niños mayor actividad en clase y por consiguiente mejor rendimiento académico.

En resumen, este trabajo aborda el proceso coercitivo desde la perspectiva de síntesis de desarrollo, porque se integran niveles de análisis adyacentes que permiten comprender la organización del comportamiento de los niños identificados como coercitivos, en su contexto específico y de forma sistemática. Se contemplan los eventos disposicionales del comportamiento coercitivo, la motivación de los niños y la red social en la que están inmersos, ya que tales eventos pueden inhibir, facilitar o acelerar el comportamiento coercitivo en los niños. Por ello, se debe considerar la organización del comportamiento de cada niño, porque las implicaciones en la forma de relacionarse con los otros, y en la solución de conflictos es distinta.

Aunque el tamaño de la muestra es pequeño, las implicaciones en la propuesta metodológica y conceptual son estimulantes. Por ello, es necesario investigar en trabajos futuros otros mecanismos adicionales que pueden aplicarse a programas de prevención y tratamiento de interacciones coercitivas en el escenario escolar. Por ejemplo, se requiere de un análisis más detallado de las consecuencias específicas que los niños reciben por su comportamiento académico o pro-social en relación al recibido por su comportamiento coercitivo, de su vinculación con pares específicos y como estos contribuyen directamente al mantenimiento o inhibición de los patrones coercitivos, y también de cómo los pares pueden ser entrenados para responder asertivamente a las iniciativas coercitivas de los niños Riesgo.

Así se podrá tener mayor impacto en el desarrollo social y académico de los niños. Además, convendrá realizar estudios longitudinales que faciliten un seguimiento más extenso en tiempo.

\section{REFERENCIAS}

Bakeman, R. \& Gottman, J.M. (1986). Observación de la interacción: Introducción al análisis secuencial. Madrid: Morata.

Bijou, S. W., \& Baer, D. M. (1975). Psicología del desarrollo infantil. México: Trillas.

Boivin, M., \& Bègin, G. (1989). Peer status and self-perception among early elementary school children. Child Development, 60, 591-596.

Cairns, R. B. (1979) Social development: The origins and plasticity of interchanges. San Francisco: Freeman. 
Cairns, R., Bergman L., \& Kagan J. (1998). Lawfulness in individual development. En R. B. Cairns, L. R. Bergman, \& J. Kagan (Eds.) Methods and models for studying the individual, (pp. 1-9), Thousand Oaks, Calif.: SAGE Publications.

Cairns, R. B. \& Cairns, B. D. (1994) Social Lifelines and Risk: Pathways of youth in our time. Cambridge: Cambridge University Press.

Cairns, R. B., Cairns, B. D., Neckerman, H. J. Gest, S. D., \& Garièpy, J. (1988) Social Network and aggressive behavior: peer support or peer rejection. Developmental Psychology, 24, 815-823.

Cairns, R. B., Santoyo, V. C., \& Holly, K. A. (1994). Aggressive escalation: Toward a development analysis. En M. Potegal \& J. Knutson (Eds.). Escalation of aggression: Biological and Social Processes (pp. 227-253). Hillsdale, N.J.:Lawrence Erlbaum Associates.

Chang L. (2003) Variable effects of children's aggression, social withdrawal, and prosocial leadership as functions of teacher beliefs and behaviors. Child Development, 74, 2, 535-548.

Chen, X., Li, D. \& Rubin, K. H. (1997) Relation between Academic Achievement and Social Adjustment: Evidence from Chinese children. Developmental Psychology, 33, 3, 518-525.

Cillessen, A. H., van ljzendoorn, H. W., van Lieshout C. F., \& Hartup, W. W. (1992). Heterogeneity among peer rejected boys: Subtypes and stabilities. Child Development, 63, 893-905.

Coie, J. D., \& Dodge, K. A. (1998). Aggression and antisocial behavior. En W. Damon, \& R. Lerner (Eds.) Handbook of child psychology. Fifth Edition. (pp. 780-850). New York: John Wiley and Son INC.

Dodge, K. A, \& Frame, C. L. (1982). Social cognitive biases and deficits in aggressive boys. Child Development, 53, 620-635.

Estell, D. B. Cairns, R. B., Farmer, T. W \& Cairns, B. D. (2002) Aggression in innercity early elementary classrooms: individual and peer group configurations. Merrill Palmer Quarterly, 48, 1, 52-76.

Farmer, T. W., \& Cairns, R. B. (1991). Social network and social status in emotionally disturbed children. Behavioral Disorders, 16, 4, 288-298.

French, D. (1988). Heterogeneity of peer-rejected boys: aggressive and nonaggressive subtypes. Child Development, 59, 976-985.

González N. M. F. (1998) Estudio de los patrones conductuales de niños agresivos: Un enfoque contextual. Tesis de Licenciatura en Psicología, Universidad Nacional Autónoma de México.

Juvonen, J., \& Wentzel, K. R. (2001) Motivación y adaptación escolar. México:Oxford University Express.

Ladd, G. W., \& Burgess, K. B. (1999). Charting the relationship trajectories of aggressive, withdrawn and aggressive/withdrawal children during early grade school. Child Development, 70, 910-929.

López, R. F. (1984). Sistemas educativos y modelos motivacionales: Delimitación de un marco de referencia. Revista de Análisis del Comportamiento, 2, 105-118.

Maciel A. O. (1996) Estabilidad y cambio de la conducta agresiva: un estudio longitudinal. Tesis de Licenciatura en Psicología, Universidad Nacional Autónoma de México. 
Magnusson, D. (1998) The logic and implications of a person-oriented approach. En R. B. Cairns, L. R. Bergman, \& J. Kagan (Eds.). Methods and models for studying the individual, (pp. 33-64), Thousand Oaks, Calif.: SAGE Publications.

Magnusson, D., \& Stattin, H. (1998). Person-context interaction theories. En W. Damon, \& R. Lerner (Eds.) Handbook of Child Psychology. Fifth Edition. Vol 1. Social, emotional, and personality development. (pp. 685-760), New York: John Wiley and Son INC.

Mayer, G. R. (1995) Preventing antisocial behavior in the schools. Journal of applied behavior analysis, 28, 467-478.

Neckerman, H. J. (1996). The stability of social groups in childhood and adolescence: The role of the classroom social environment. Social Development, 5, 2.

Olweus, D. (1979). Stability of aggressive reaction patterns in males: A review. Psychological Bulletin, 86, 852-875.

Oñate, M. P. (1989). El auto concepto. Madrid :Narcea, S.A de Ediciones Madrid.

Patterson, G. R. (1974) A basis for identifying stimuli which control behaviors in natural settings. Child Development, 45, 900-911.

Patterson, G. R. (1982) Coercive family process: A social learning approach. Vol. III. Eugene, Oregon: Castalia Publishing Company.

Patterson, C. J., Kupersmidt, J. B., \& Griesler, P. C.(1990). Children's perceptions of self and relationships with others as a funtion of sociometric status. Child Development, 61, 1335-1349.

Rubio, M. P. (2004) Redes sociales establecidas en aulas integradoras: la perspectiva de los docentes: la perspectiva de los docentes y los alumnos. Tesis de Doctorado en Psicología, Universidad Nacional Autónoma de México.

Rubio, M. P., \& Santoyo, V. C. (2004) Interacciones sociales de niños con necesidades educativas especiales: Un enfoque funcional. Revista Mexicana de Análisis de la Conducta, 30, 1, 1-20.

Santoyo, V. C. (1997). Sobre el desarrollo de la conducta social. Revista de Psicología Contemporánea, 4, 4, 38-45.

Santoyo, V. C., Colmenares, V. L., \& Losada, L. J.L, (2007). Redes sociales: modalidades, funcionalidad y continuidad en una disciplina de síntesis. En C. Santoyo (Ed.). Estabilidad y Cambio de Patrones de Comportamiento en escenarios naturales: un estudio longitudinal en Coyoacan (pp. 81-112). México: UNAM/CONACYT.

Santoyo, V. C., Espinosa, M. C., \& Bachá, M., D. (1994). Extensión del sistema de observación conductual de las interacciones sociales: calidad, dirección, contenido, contexto y resolución. Revista Mexicana de Psicología, 11, 1, 55-68.

Santoyo, V. C., Espinosa, M. C., \& Maciel, A. O. (1996). Reciprocidad y patrones coercitivos de niños preescolares. Revista Mexicana de Análisis de la Conducta, 13, 1, 63-74.

Santoyo, V. C., \& Rubio, M. P. (2006). Desarrollo y función del comportamiento autodescriptivo: implicaciones metodológicas y conceptuales desde una perspectiva de síntesis. En C. Santoyo y M. C. Espinosa (Eds.). Desarrollo e interacción social: Teoría y métodos de investigación en contexto (pp. 81-111). México: UNAM/ CONACYT. 
Sidman, M. (1989) Coercion and its fallout. Boston, Mass: Authors Cooperative, Inc. Wilson, J. Q., \& Herrnstein, R. J. (1985) Crime and human nature. New York: Simon and Shuster.

\section{APÉNDICE 1}

Definición de las categorías del Sistema de Observación Conductual de las Interacciones Sociales, SOC-IS, utilizadas en el estudio (Santoyo, Espinosa y Bachá, 1994).

\begin{tabular}{|c|c|c|}
\hline $\begin{array}{l}\text { Categoría } \\
\text { Conductual }\end{array}$ & Código & Definición \\
\hline $\begin{array}{l}\text { Emisión del sujeto } \\
\text { focal (SF) }\end{array}$ & & $\begin{array}{l}\text { Conducta física y/o verbal que el SF dirige a } \\
\text { otro(s) niño(s), sin que en el intervalo prece- } \\
\text { dente haya existido iniciativa de esta acción } \\
\text { por parte de otro(s) hacia el SF. Se anotará el } \\
\text { o los nombre(s) de quien(es) recibe(n) la ac- } \\
\text { ción del SF. }\end{array}$ \\
\hline $\begin{array}{l}\text { Emisión Negativa } \\
\text { del SF }\end{array}$ & & $\begin{array}{l}\text { Conducta física y/o verbal de tipo coercitivo } \\
\text { que el SF dirige a otro(s) niño(s), sin que en el } \\
\text { intervalo precedente haya existido la iniciativa } \\
\text { de esta acción por parte de otro(s) hacia el SF, } \\
\text { anotándose el (los) nombre(s) de quien(es) } \\
\text { recibe(n) la acción negativa (evento aversivo } \\
\text { o punitivo del SF. }\end{array}$ \\
\hline $\begin{array}{l}\text { Recepción hacia } \\
\text { el SF }\end{array}$ & & $\begin{array}{l}\text { Conducta física y/o verbal iniciada por otro(s) } \\
\text { niño(s) y dirigidas hacia el SF; sin que se pre- } \\
\text { sente en el intervalo inmediato anterior o en el } \\
\text { mismo, alguna iniciación por parte del SF. }\end{array}$ \\
\hline $\begin{array}{l}\text { Recepción } \\
\text { Negativa hacia } \\
\text { el SF }\end{array}$ & & $\begin{array}{l}\text { Conducta física y/o verbal de tipo coercitivo } \\
\text { (aversivas o punitivas) iniciada por otro(s) } \\
\text { niño(s) y dirigidas hacia el SF; sin que en el in- } \\
\text { tervalo inmediato anterior o en el mismo haya } \\
\text { existido iniciación o respuesta del SF. }\end{array}$ \\
\hline Interacción Social & S & $\begin{array}{l}\text { Conductas físicas y/o verbales del SF y/o } \\
\text { compañero(s) dirigidas hacia el o los otro(s) } \\
\text { (niños), simultáneas o sucesivas, en las que } \\
\text { existe dependencia mutua. Se incluyen con- } \\
\text { ductas de asentimiento o negación con mo- } \\
\text { vimientos de cabeza y/o extremidades de los } \\
\text { participantes. }\end{array}$ \\
\hline
\end{tabular}




\begin{tabular}{lcl}
\hline $\begin{array}{l}\text { Categoría } \\
\text { Conductual }\end{array}$ & Código & \multicolumn{1}{c}{ Definición } \\
\hline $\begin{array}{l}\text { Interacción Social } \\
\text { Negativa }\end{array}$ & S-- & $\begin{array}{l}\text { Conductas físicas y/o verbales (de contacto } \\
\text { físico), de tipo coercitivo del SF o el compa- } \\
\text { ñero dirigidas hacia el otro, llevadas a cabo de } \\
\text { forma simultánea o sucesiva, existiendo de- } \\
\text { pendencia mutua entre los participantes de la } \\
\text { interacción. Se incluyen aquí conductas como } \\
\text { amenazas, insultos, intimidación, golpes, pa- } \\
\text { tadas, empujones. }\end{array}$ \\
$\begin{array}{lll}\text { Actividad } & \text { Conductas realizadas por el sujeto focal por } \\
\text { instrucción expresa de su profesor y acorde } & \text { con su objetivo instruccional vigente. Implica } \\
\text { contacto y uso del material, según los criterios } \\
\text { de ejecución de la tarea. }\end{array}$ \\
Otra(s) & OR & $\begin{array}{l}\text { Se define como todas aquellas conductas que } \\
\text { realiza el SF y que no están englobadas en las } \\
\text { demás actividades. }\end{array}$ \\
\hline
\end{tabular}

